\title{
EXPERIENCIA DE PERSONAS CON CÁNCER QUE REALIZAN TERAPIA COMPLEMENTARIA: REVISIÓN INTEGRATIVA
}

\author{
EXPERIENCE OF PEOPLE WITH CANCER WHO PERFORM \\ COMPLEMENTARY THERAPY: INTEGRATIVE REVIEW
}

\section{EXPERIÊNCIA DE PESSOAS COM CÂNCER QUE REALIZAM TERAPIA COMPLEMENTAR: REVISÃO INTEGRATIVA}

\author{
Sophia Herrera Contreras* \\ Maritza Espinoza Venegas** \\ Julia Huaiquian Silva***
}

\begin{abstract}
RESUMEN
Objetivo: Identificar evidencia científica relacionada con la experiencia de pacientes oncológicos que practican terapias complementarias. Material y Método: Revisión integrativa que siguió los 5 pasos metodológicos de Crossetti, cuya búsqueda bibliográfica, considerando los términos "Terapias Complementarias", "Neoplasia”, "Enfermería", "Experiencia e Investigación Cualitativa”, en portugués e inglés y utilizando los operadores booleanos "AND" y "OR", se realizó en las bases de datos BVS, SCIELO, SCOPUS y WOS, publicada entre los ańos 2000 y 2019. Resultados: 16 artículos primarios de tipo cualitativo cumplieron criterios de inclusión. Se identificaron 6 categorías: creencias sobre las terapias complementarias, terapia complementaria v/s terapia convencional, cuidado holístico del ser, tiempo para el amor personal, dar sentido a la experiencia del cáncer y aumento del control personal. Conclusiones: Las personas con cáncer que utilizan terapias complementarias experimentan vivencias positivas, de buena calidad de vida, con beneficios sobre los síntomas propios del cáncer o de la quimioterapia y mejoras en la salud mental, espiritual y social. Los estudios cualitativos sobre las terapias complementarias se centran en las razones que llevan a los pacientes a utilizarlas, sus creencias y la satisfacción de necesidades como un todo. Es necesario indagar dichas experiencias en terapias específicas y reconocer cuál es más beneficiosa para una persona con cáncer. Más evidencia podría justificar su incorporación a los cuidados formales de la práctica de Enfermería.
\end{abstract}

Palabras clave: Terapias Complementarias; Neoplasias; Enfermería oncológica; Experiencias de vida; Investigación Cualitativa.

\footnotetext{
*Magíster (c). Programa Magíster en Enfermería, Facultad de Enfermería, Universidad de Concepción. ORCID: https://orcid. org/0000-0002-9998-9060. Email: sophiaherrerac@gmail.com.

**Doctora en Enfermería. Departamento Materno Infantil, Facultad de Enfermería, Universidad de Concepción. Chile. ORCID: https://orcid.org/0000-0002-2361-211X. Autor por correspondencia: Email: mespinoz@udec.cl.

****Doctora en Enfermería. Departamento Materno Infantil, Facultad de Enfermería, Universidad de Concepción. Chile. ORCID: https://orcid.org/0000-0002-0415-1848. Email: jhuaiqui@udec.cl.
} 


\section{ABSTRACT}

Objective: To identify the scientific evidence related to the experience of cancer patients undergoing complementary therapies. Materials and Methods: Integrative review that followed Crossetti's 5 methodological steps. The search strategy considered the terms "Complementary Therapies", "Neoplasia", "Nursing", "Experience and Qualitative Research", using the Boolean operators "AND" and "OR" in Spanish, Portuguese and English. It was carried out in the BVS, SCIELO, SCOPUS, WOS data bases, in the period between 2000 and 2019. Results: 16 qualitative primary articles met the inclusion criteria. Six categories were identified: beliefs about complementary therapies, complementary therapy vs. conventional therapy, holistic wellbeing, time for personal love, making sense of the cancer experience and increasing personal control. Conclusions: People with cancer who use complementary therapies report positive experiences and good quality of life, with benefits over cancer or chemotherapy symptoms, and improvements in mental, spiritual and social health. Qualitative studies on complementary therapies focus on the reasons leading patients to use them, their beliefs and overall satisfaction as a whole. It is necessary to investigate these experiences focusing on specific therapies, and to recognize which is more beneficial to a person with cancer. More evidence could support its inclusion into nursing care practice.

Key words: Complementary Therapies; Neoplasms; Oncology Nursing; Life Experiences; Events, Qualitative Research.

\section{RESUMO}

Objetivo: Identificar a evidência científica relacionada à experiência de pacientes oncológicos que utilizam terapias complementares. Material e Método: Revisáo integrativa que segue as 5 etapas metodológicas de Crosseti, cuja estratégia de busca considera os termos "Terapias Complementares", "Neoplasia", "Enfermagem", "Experiência e pesquisa qualitativa", com os operadores booleanos "AND" e "OR" em espanhol, português e inglês. A busca foi realizada nas bases de dados BVS, SCIELO, SCOPUS e WOS, no período compreendido entre os anos 2000 e 2019. Resultados: 16 artigos primários do tipo qualitativo cumpriram critérios de inclusão. Foram identificadas 6 categorias: crenças sobre as terapias complementares, terapia complementar versus terapia convencional, cuidado holístico do ser, tempo para o amor pessoal, dar sentido à experiencia do câncer e aumento do controle pessoal. Conclusóes: As pessoas com câncer que utilizam terapias complementares têm experiências positivas, boa qualidade de vida, com benefícios sobre os sintomas do câncer ou da quimioterapia e melhorias na saúde mental, espiritual e social. Os estudos qualitativos sobre as terapias complementares estáo enfocados nas razóes que levam aos pacientes a utilizá-las, suas crenças e a satisfaçáo de necessidades como um todo. É necessário investigar essas experiências em terapias específicas e reconhecer qual entrega mais benefícios a uma pessoa com câncer. Mais evidências poderiam justificar a incorporação aos cuidados formais da prática de Enfermagem.

Palavras-chave: Terapias Complementares; Neoplasias; Enfermagem oncológica; Acontecimentos que mudam a vida; Pesquisa qualitativa.

Fecha de recepción: 04/10/2019

Fecha de aprobación: 02/01/20

\section{INTRODUCCIÓN}

Actualmente el cáncer constituye una de las primeras causas de muerte a nivel mundial ${ }^{(1)}$. En Chile la situación no es distinta, dado que el cáncer es la segunda causa de muerte de la población chilena, luego de las afecciones al sistema circulatorio y cardiovascular, proyectándose que al final de la próxima década llegue a ser la primera causa de muerte en el país ${ }^{(2)}$. El cáncer representa un padecimiento físico y un estado de ansiedad ante los diferentes cambios que se generan a partir del diagnóstico, el tratamiento prolongado y los efectos asociados. Esto implica una interrupción 
biográfica y una incesante búsqueda de respuestas que lleva a la persona y su familia a aferrarse a diversas estrategias, prácticas y tratamientos que en algunas ocasiones pueden diferir de lo que ofrece la medicina convencional ${ }^{(3)}$.

En este mismo sentido, y teniendo en cuenta la necesidad de un cuidado integral que abarque dimensiones no sólo físicas y biológicas, sino también emocionales, mentales y espirituales, la Organización Mundial de la Salud (OMS), actualmente reconoce los aportes de la medicina alternativa y/o medicina complementaria y la define como aquellas prácticas, enfoques, conocimientos y diversas creencias sanitarias que incorporan medicinas basadas en plantas, animales y/o minerales, terapias espirituales, técnicas manuales y ejercicios aplicados de forma individual o en combinación para mantener el bienestar, además de tratar, diagnosticar y prevenir las enfermedades ${ }^{(4)}$.

Según un estudio de percepción social en España ${ }^{(5)}$, las terapias complementarias utilizadas con mayor frecuencia son: yoga $(32,5 \%)$, acupuntura/medicina tradicional china $(31,5 \%)$, quiromasaje $(28,4 \%)$ y homeopatía $(23 \%)$ y, en menores porcentajes, la reflexología podal, tai-chi, terapia floral, drenaje linfático, naturopatía, reiki, osteopatía y shiatsu. Por su parte en Chile ${ }^{(6)}$, el Ministerio de Salud (MINSAL) reconoce que las terapias complementarias que tienen una mayor oferta en el país, son la naturopatía, la homeopatía, la acupuntura, las terapias florales, el reiki y la aromaterapia, entre otras.

Las Terapias Complementarias y Alternativas (TCA) están siendo cada vez más utilizadas por personas en países occidentales después de un diagnóstico de cáncer. La prevalencia de su uso en adultos con diagnóstico de cáncer tiene un promedio de entre 30 y $40 \%$ en Europa y Norteamérica $^{(7)}$. Estas terapias se administran en varios contextos terapéuticos, ya sea como una entidad independiente de la atención convencional (medicina alternativa), junto con la atención médica estándar (medicina complementaria), o como una parte integral de la atención convencional contra el cáncer (oncología integradora) ${ }^{(8)}$.

Los pacientes con cáncer usan estas terapias tanto para paliar efectos secundarios de los tratamientos, o como recurso de afrontamiento psicológico, pues les ayuda a sentir que tienen más control de su situación y mantener una actitud más positiva, de esperanza frente a todo lo adverso que puede resultar una enfermedad oncológica ${ }^{(8)}$. Otras investigaciones $^{(9,10)}$ evidencian que los pacientes recurren a la TCA, en parte, como resultado de la insatisfacción con la atención convencional y que, en general, son las mujeres quienes usan más estas terapias. La literatura también informa sobre los efectos beneficiosos de las terapias complementarias $^{(11)}$, aun cuando la mayoría de ellos se centran en la medición de resultados preestablecidos, como la calidad de vida y otros ${ }^{(12)}$. También se observa que los resultados obtenidos difieren en su efectividad pues, mientras algunos evidencian aumento de calidad de vida al utilizar estas terapias ${ }^{(13)}$, otros no demuestran un efecto significativo $^{(14,15)}$. Quizás el abordaje cuantitativo, de muchos estudios, impide medir aspectos subjetivos en profundidad, como la experiencia de usar estas terapias y sus beneficios en el ámbito espiritual. En este sentido, destacan investigaciones que detallan experiencias y percepciones de mejor manejo de sentimientos como el miedo, la ansiedad, o síntomas como el dolor, vómitos y náuseas, además de un aumento de los sentimientos de control, relajación, felicidad y conciencia de sí mismo ${ }^{(16)}$.

Considerando que los estudios cualitativos aportan con una comprensión más profunda y personal de las personas y que Enfermería debe responder a las necesidades que surgen de los cuidados integrales de sus pacientes, a partir de un diálogo abierto y reconociendo la necesidad de integrar las terapias complementarias al quehacer de enfermería, el objetivo de este estudio fue identificar la evidencia relacionada con la experiencia de las personas con cáncer que practican terapias complementarias. Según lo anterior, la pregunta guía de esta revisión fue: ¿Cuál es la evidencia científica de enfermería relacionada con la experiencia de las personas con cáncer que practican terapias complementarias?

\section{MATERIAL Y MÉTODO}

Se realizó una revisión integrativa de la evidencia científica bajo las recomendaciones de Crossetti ${ }^{(17)}$, que sigue básicamente cinco etapas: 1) formulación del problema y pregunta de investigación, 2) búsqueda de la literatura, 3) evaluación de los artículos, 
4) síntesis de los artículos y 5) interpretación de los resultados o discusión.

La búsqueda fue llevada a cabo en el año 2019 en bases de datos: BVS, SCIELO, SCOPUS y WOS. La estrategia de búsqueda consideró las palabras claves: Terapias Complementarias, Neoplasia, Enfermería, Experiencia e Investigación Cualitativa con su respectiva traducción en inglés (Complementary Therapies, Neoplasms, Nursing, Experience y Qualitative Research) y portugués (Terapias Complementares, Neoplasia, Enfermagem, Experiência y Pesquisa Qualitativa). Los operadores booleanos utilizados fueron "AND" y "OR" y los criterios de inclusión correspondieron a: investigaciones primarias de tipo cualitativas, realizadas por profesionales de enfermería, a texto completo disponible en línea, en el idioma inglés o español o portugués, publicados entre los años 2000 al 2019. Se excluyeron revisiones, tesis, capítulo de libro y editoriales. Se identificaron 118 artículos, de los cuales debieron excluirse los que no tenían acceso a texto completo, los que se encontraron duplicados en la medida que se avanzaba en la recuperación en las bases de datos y los que no cumplieron con la rigurosidad metodológica según Gálvez $^{(18)}$ (Figura 1). Del análisis exhaustivo se seleccionaron 16 artículos ${ }^{(19-34)}$, que cumplieron los criterios de inclusión (Tabla 1). Sus resultados fueron interpretados mediante el análisis de contenido ${ }^{(35)}$ y se identificaron las categorías referidas por los autores, sus similitudes y diferencias.

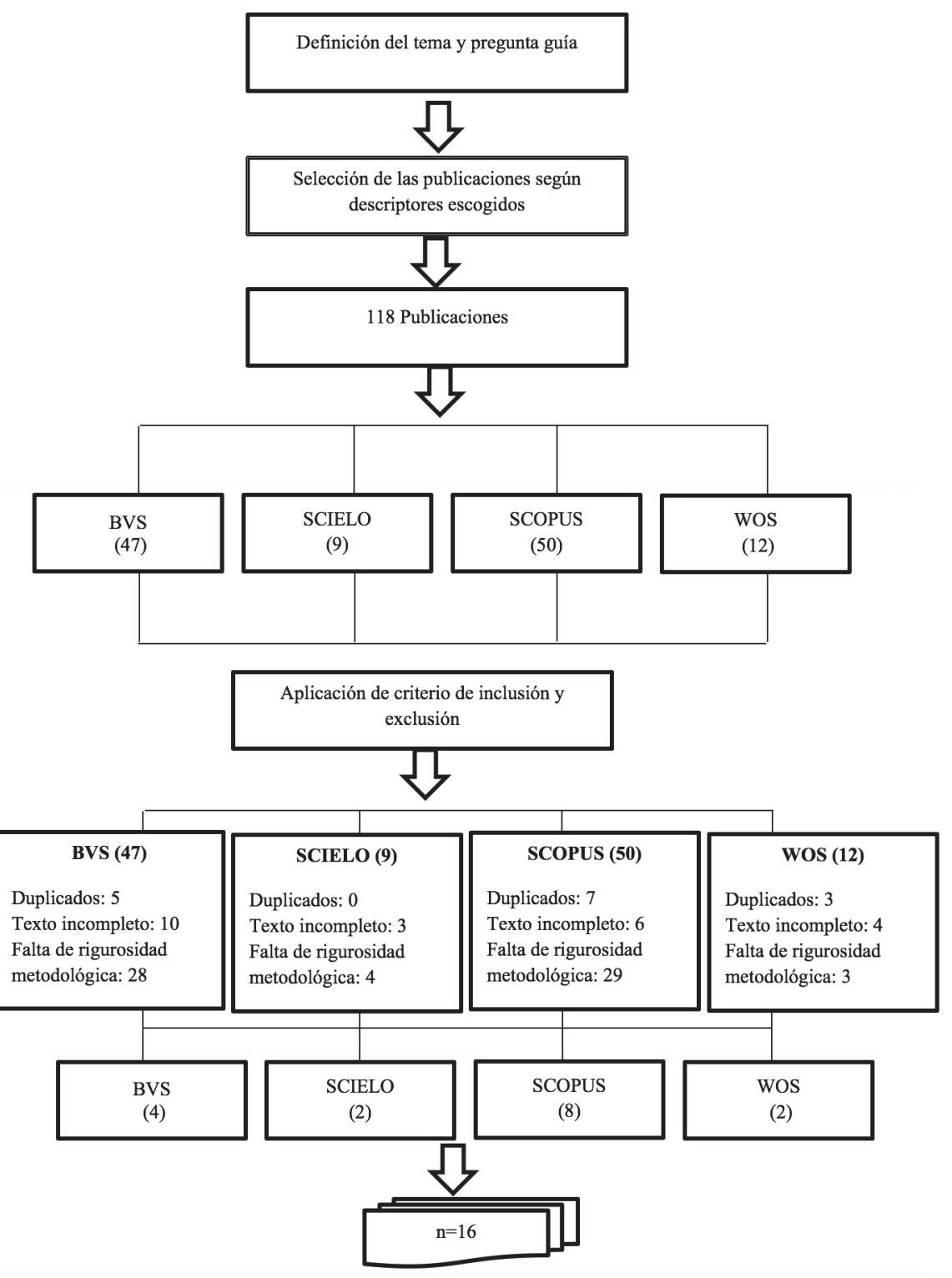

Figura 1. Diagrama de flujo de identificación, selección e inclusión de los estudios. 


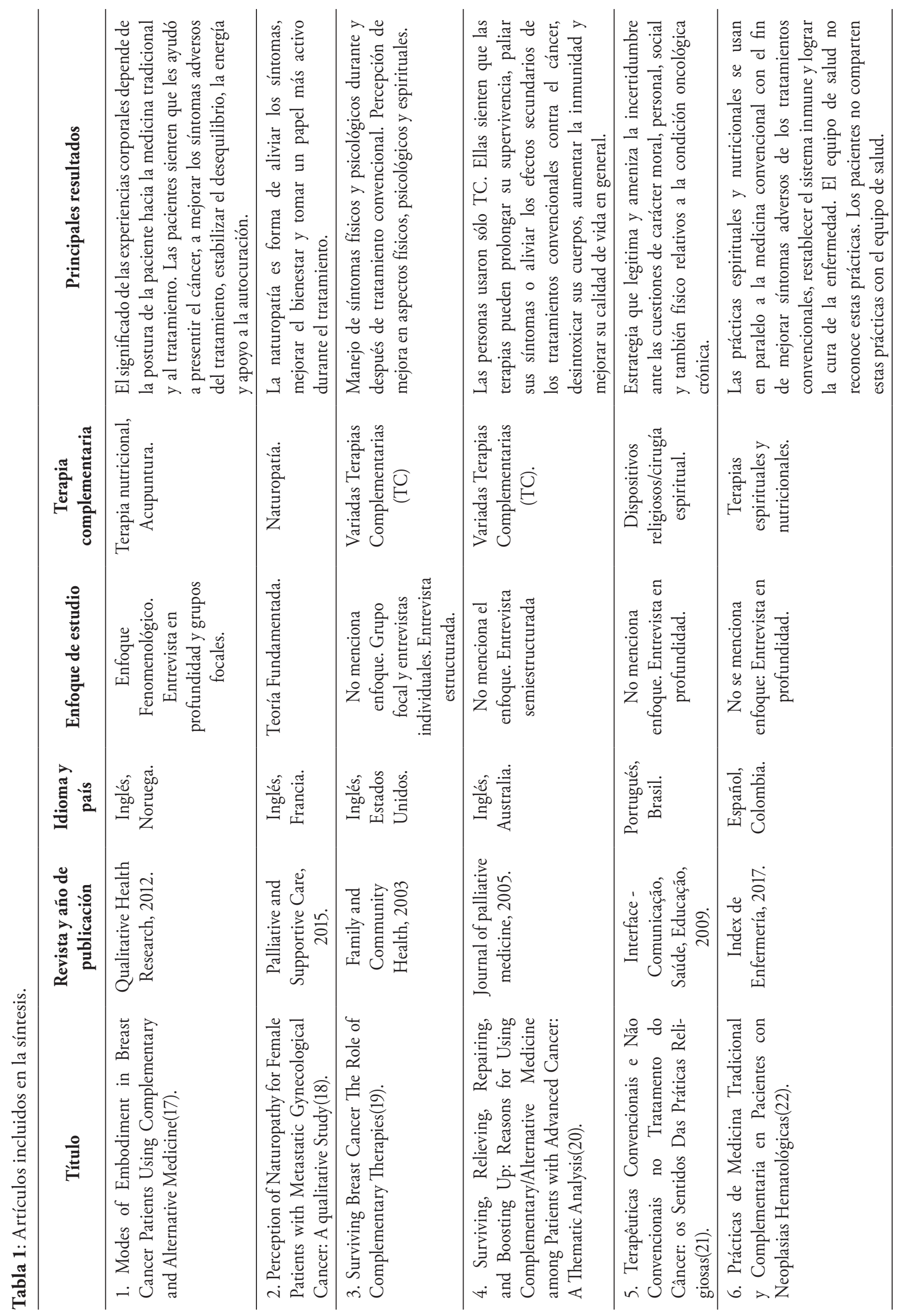




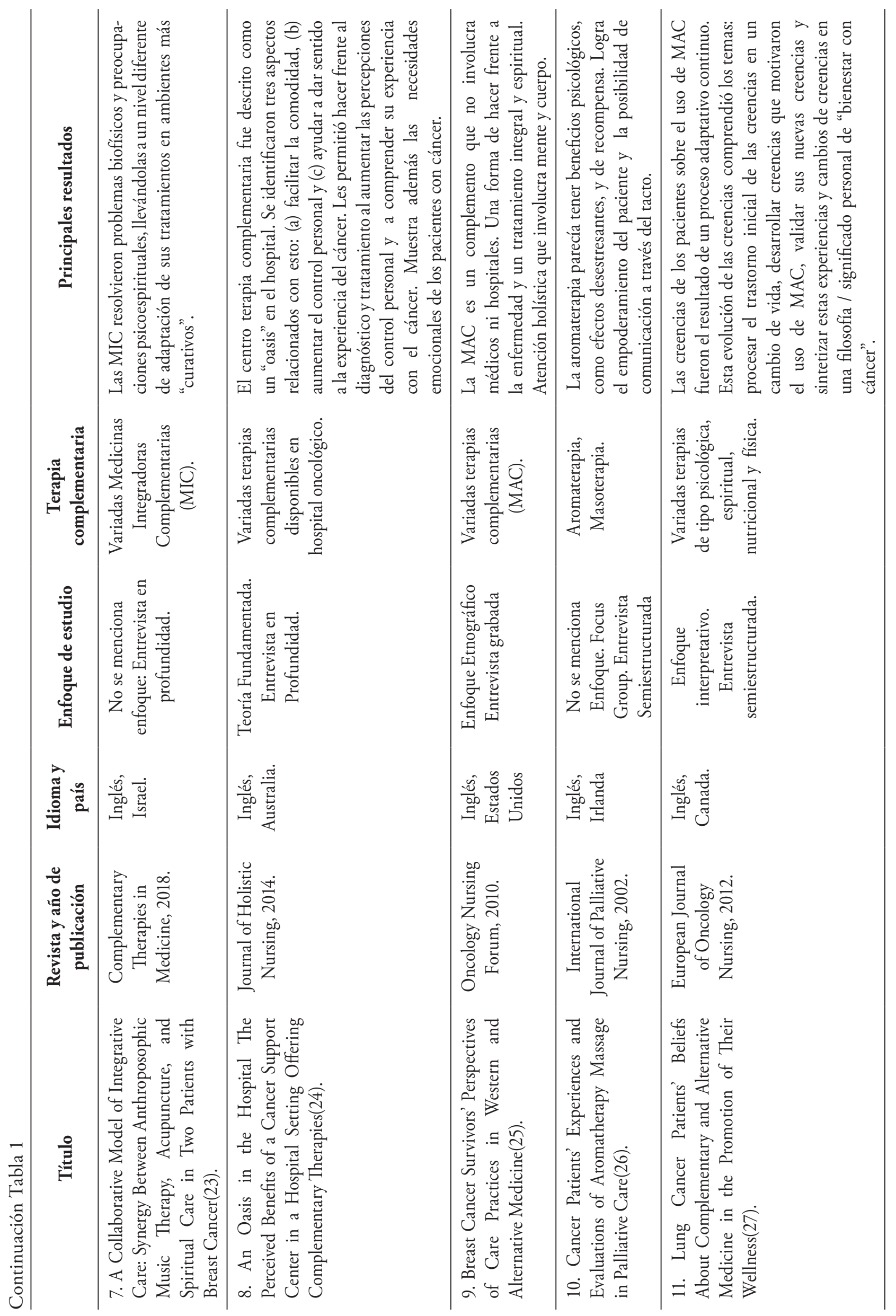




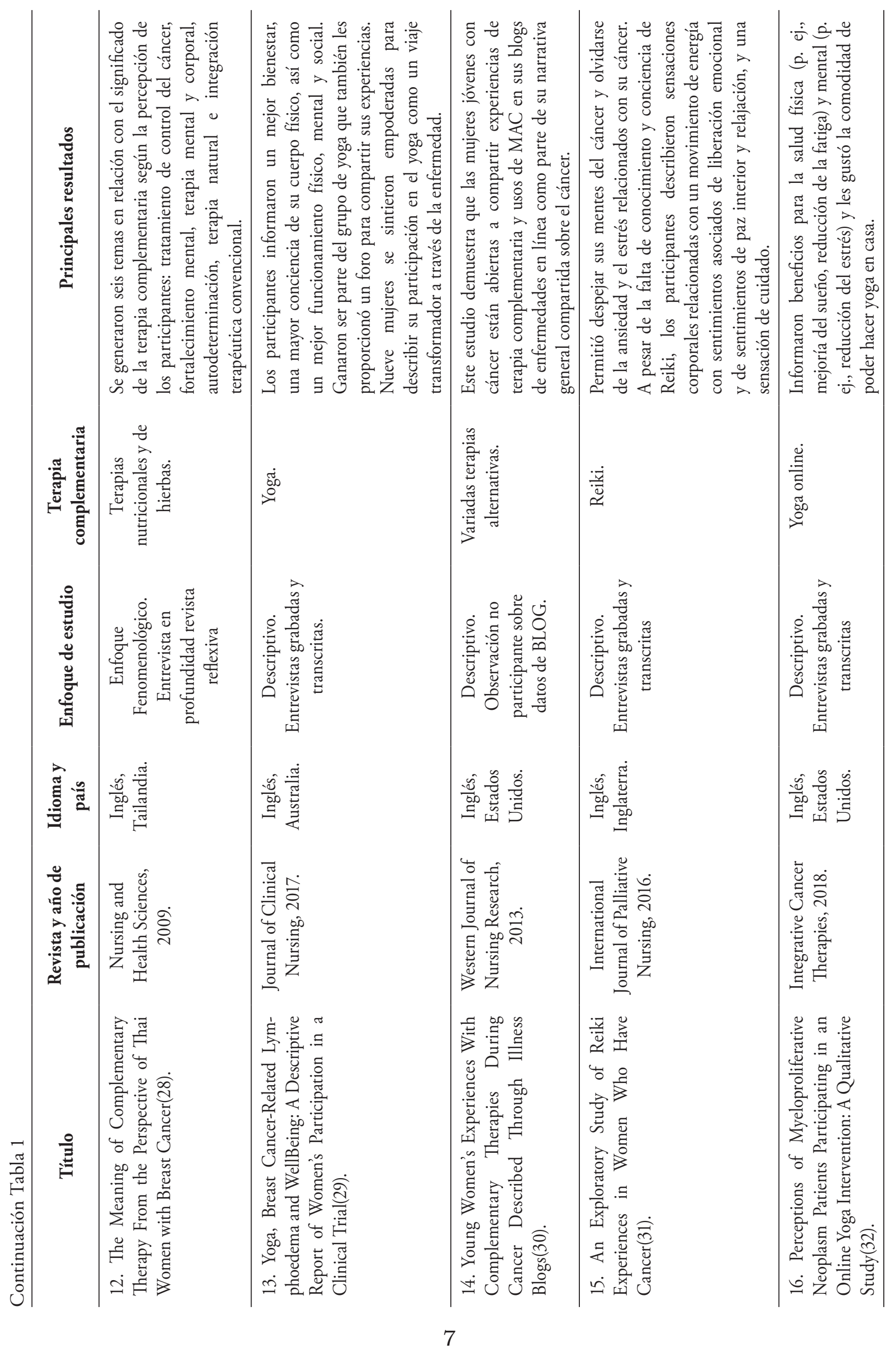




\section{RESULTADOS}

La mayoría de los artículos seleccionados (10) fueron publicados entre los años 2011 y 2019, en idioma inglés preferentemente (14); las terapias complementarias o alternativas fueron variadas y la mitad de los estudios presentaban terapias combinadas; el número de participantes de cada estudio osciló entre 2 a 66; 9 estuvieron dirigidos a mujeres con cáncer y el resto a personas de ambos sexos (Tabla 1). De este análisis emergieron 6 categorías y 6 subcategorías (Figura 2):

Creencias sobre las Terapias Complementarias/ Alternativas. En esta categoría se pudieron rescatar dos subcategorías: Prejuicios y Beneficios. 1) Respecto a los Prejuicios, los participantes expresan diversos prejuicios respecto a las TCA. En la mayoría de los casos mencionan que no son reconocidas por la biomedicina y que las autoridades médicas subestiman el valor de lo complementario y alternativo y que algunos sugieren, además, que es una pérdida de tiempo y dinero ${ }^{(27,31)}$. Lo anterior influye de manera significativa en la percepción que tienen los pacientes sobre la utilización de estas terapias $^{(29)}$. Otro aspecto mencionado es la reticencia a contarle al resto de las personas que practican dichas terapias, porque temen ser ridiculizados y sentirse vulnerable a los juicios de $\operatorname{otros}^{(28,33,34)}$. 2) Respecto a los Beneficios, uno de los principales motivos para el uso de TCA es la creencia que estas mejoran el sistema inmune y linfático, ralentizan el crecimiento del cáncer y desintoxican el cuerpo eliminando toxinas que provienen del cáncer o la quimioterapia ${ }^{(21,22,25,}$ 30). Otras creencias se relacionaban con que las terapias complementarias alivian los síntomas del cáncer, aumentan la sobrevivencia y, por lo tanto, prolongan la vida ${ }^{(22,32)}$. Además, se menciona que los productos utilizados en las terapias provienen de la naturaleza, por lo que no hay contaminantes ni toxinas, que son de fácil acceso, simples e indoloros. Los pacientes validan sus creencias sobre las terapias complementarias a través de un proceso de prueba y error, es decir, cuando éstos sienten los efectos tangibles del tratamiento, sus creencias son reforzadas ${ }^{(24,29)}$.

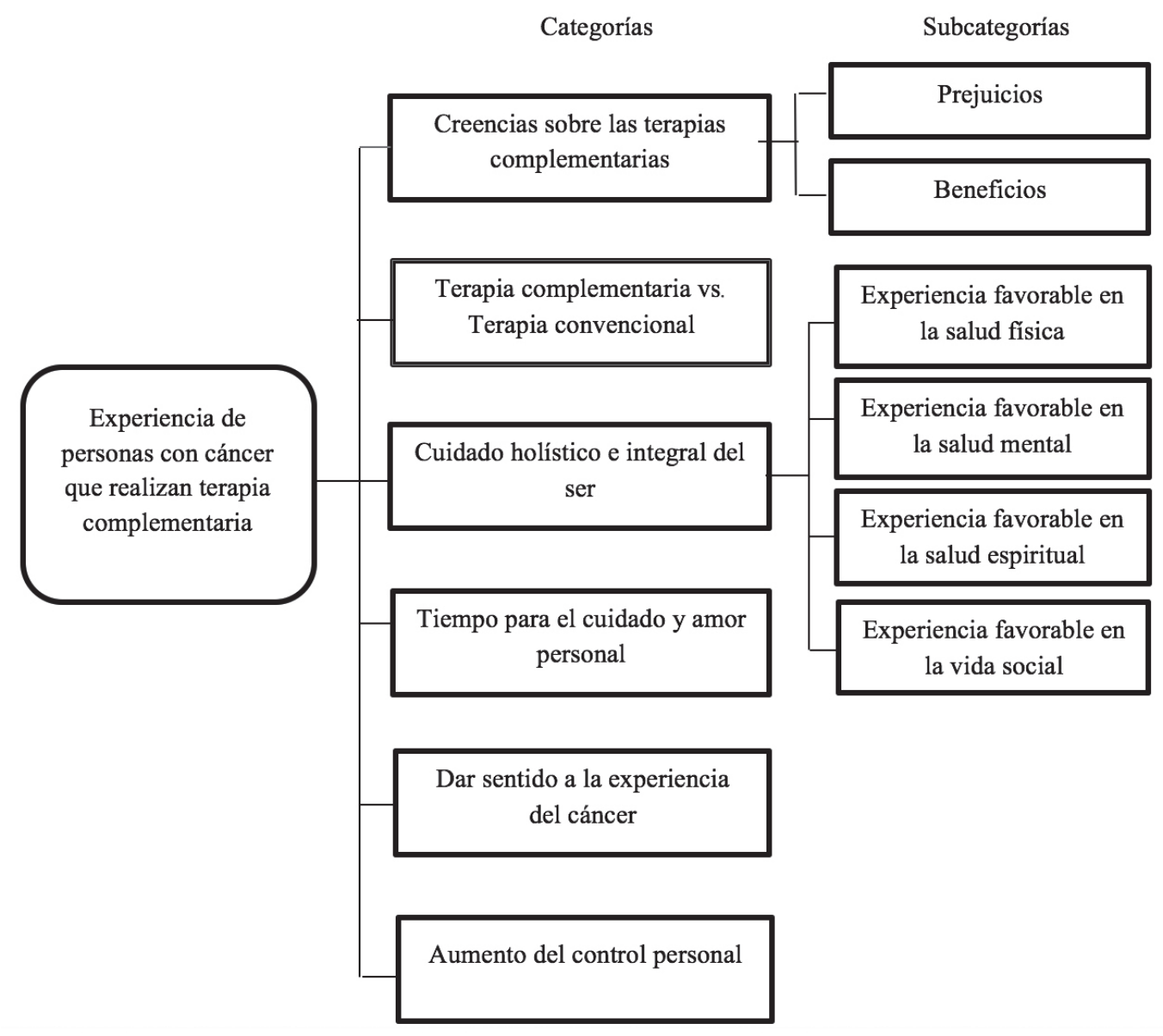

Figura 2. Experiencia de personas con cáncer que realizan terapia complementaria. 
Terapia complementaria vs. Terapia convencional. La terapia convencional se centra en el cuerpo y la enfermedad, dejando de lado muchas veces las otras dimensiones de la persona, como la mente y el espíritu, por lo que, a la vista de los pacientes, existe un vacío en la atención biomédica ${ }^{(20,22,23,26)}$. Estos mismos manifiestan que utilizan TCA cuando la medicina convencional no responde a sus demandas y en la mayoría de los casos son usadas en paralelo con las quimioterapias ${ }^{(21)}$. El cuidado convencional se siente más completo cuando está acompañado de la terapia complementaria, ya que esta última responde a las necesidades individuales y no es solo una receta para el tratamiento del cáncer ${ }^{(30)}$.

Cuidado holístico e integral del ser. En general la medicina tiende a atender sólo una parte de la persona, pasando por alto la complejidad que representa el ser humano ${ }^{(31,32)}$. El cuidado holístico e integral del ser está dirigido a armonizar cuerpo, mente, alma y emociones, para que de esta manera se logre un equilibrio que apunte hacia un estado de bienestar y a una mejor calidad de vida ${ }^{(19,20,27,29,30)}$. Esto se evidencia por los relatos de las experiencias favorables del ser que genera el TCA, descritas a continuación en 4 subcategorías: 1) Experiencia favorable en la salud física: Las TCA actúan sobre los síntomas propios del cáncer y los síntomas secundarios al tratamiento convencional ${ }^{(27,30,31,}$ 33). Los participantes notaron cambios físicos que iban desde la reducción del dolor, hinchazón y úlceras, disminución de lesiones en la piel, vómitos y hormigueos, hasta la mejoría de la calidad del cabello, uñas o la circulación y fortalecimiento de la condición física en general ${ }^{(19-22,25,26,28,32,34)}$. 2) Experiencia favorable en la salud mental: Las TCA permiten expresar preocupaciones y miedos, liberando así el estrés y la tensión acumulada ${ }^{(26,28,}$ 34). Se reduce también el cansancio, la ansiedad, y las preocupaciones ${ }^{(20,22,25)}$ y esto genera un aumento del ánimo, la energía y mejoría en el patrón de sueño. Los participantes expresan sentirse relajados, felices, con sentimientos de bienestar, mencionan además que gracias a las terapias complementarias pudieron aumentar la confianza y la autoestima e incluso aliviar la depresión ${ }^{(19-21,30,31,33)}$. 3) Experiencia favorable en la salud espiritual: La oración y la meditación entrega la tranquilidad para enfrentar la enfermedad ${ }^{(27)}$, profundiza la capacidad de tocar el yo interior, entregando serenidad y calma en momentos de tempestad ${ }^{(25,30)}$. Para los participantes la espiritualidad representa una fuente de paz, comodidad y una fuerza emocional cuando hay miedo y preocupación ${ }^{(23,24)}$. 4) Experiencia favorable en la vida social: El contexto donde se lleva a cabo la TCA brinda seguridad y confianza, lo que permite compartir experiencias y crear un fuerte sentido de conexión entre compañeros ${ }^{(21)}$. Además, los participantes destacan la labor del proveedor de la terapia complementaria, quien cumple el rol de escucha activa y comprensión de sus experiencias ${ }^{(21,27,29)}$. El asistir a estas sesiones crea un sentimiento de optimismo, esperanza y aumento de autoconfianza, que permite a muchos participantes volverse más activos y mejorar las relaciones interpersonales ${ }^{(28,31)}$.

Tiempo para el cuidado y amor personal. Las sesiones de terapia complementaria son vistas como un refugio, un lugar que ofrece tranquilidad $y$ confort dentro de una vida ajetreada ${ }^{(26)}$. Otros participantes la ven como un pedacito de cielo, un momento especial y propio que sólo le pertenece a ellos $^{(26)}$. La sesión les entrega un momento para olvidar la enfermedad y sus implicaciones, un momento para reconstruir su fuerza interior y tratar de dejar atrás todo lo malo ${ }^{(28)}$. La terapia diaria les da la oportunidad de apreciar el "tiempo para uno mismo”, una hora de ser egoístas y de absorber toda esa energía positiva ${ }^{(28,31)}$.

Dar sentido a la experiencia del cáncer. La TCA entrega la oportunidad de ver al cáncer como un proceso de aprendizaje y de cambio ${ }^{(19)}$. Ayuda a que las personas comprendan lo inexplicable y puedan aceptarlo; de esta manera creer en que se puede hacer frente a la enfermedad y sobrevivir ${ }^{(22)}$. Además, da la fuerza para continuar, para poder cambiar la persona autocrítica y negativa por una persona más positiva $^{(19,21,25,30,31)}$. Las diversas modalidades de terapia logran reconectar al cuerpo de una manera más significativa, de encontrar la fuerza para ayudar a remodelar y enfocarse en la nueva identidad del $\operatorname{ser}^{(24,26,32)}$.

Aumento del control personal. Al ser libre para poder elegir con qué terapia complementaria tratar la enfermedad, seleccionarla y decidir su duración, de manera independiente y voluntaria, permite a los participantes sentirse más empoderados en tomar las riendas de la propia vida nuevamente ${ }^{(28,}$ ${ }^{29)}$. La TCA entrega la satisfacción de poder decidir y mantener un sentido de control personal, lo 
que brinda autodeterminación y aumento de confianza $^{(20,26,30,31)}$.

\section{DISCUSIÓN}

De la evidencia emergen categorías y subcategorías de experiencias en su mayoría positivas. Las creencias respecto a las TCA hacen que se muestren abiertos a utilizarlas porque comparten la idea que estos métodos son naturales, de fácil acceso, simples e indoloros ${ }^{(24,29)}$ y además, la evidencia cuantitativa $^{(36)}$ muestra que las TCA son utilizadas bajo la creencia que pueden ser beneficiosas, al mismo tiempo que inofensivas. Aun así, se describen reacciones de miedo en algunos participantes por los posibles efectos negativos que estos podrían tener o incluso que podrían interferir con el tratamiento convencional ${ }^{(37)}$. Lo anterior es comprensible porque no todas las personas poseen las mismas creencias y conocimientos $y$, en consecuencia, tienen el derecho a recibir información sobre los efectos, tanto de las terapias convencionales como complementarias y así decidir el tipo de terapia a usar.

Los beneficios más destacados en los artículos fueron la capacidad para disminuir la ansiedad, el estrés e inducir a la relajación coincidiendo con estudios que muestran similares resultados $(38,39)$ en donde las TCA han resultado efectivas para mejorar la calidad del sueño, disminuir la ansiedad, el estrés y la fatiga. Esto trae consigo, no sólo beneficios físicos, sino que además espirituales y emocionales $^{(40)}$.

Se observa además, como categoría emergente, que los usuarios integran la terapia convencional con la TCA con la idea de que no sólo es necesario tratar el cuerpo para curarse, sino también la mente, las emociones y el alma ${ }^{(41,42)}$. Así, las TCA que se señalan como un tratamiento para aliviar y curar el cáncer, también se describen como un refugio o un espacio para tener un respiro ${ }^{(43,44)}$.

El cuidado holístico e integral del ser resultó ser una categoría altamente relevante de la que subemergieron dimensiones que se constituyen como las razones para utilizar las TCA, tales como la disminución, tanto de síntomas físicos propios del cáncer, o de los efectos secundarios a la quimioterapia, como las mejoras en la salud mental, social y espiritual. Hallazgos similares ${ }^{(45,}$
46) se evidencian en estudios donde los usuarios argumentan que la enfermedad, en sí misma, tiene efectos secundarios que se contrarrestan con la terapia complementaria. Adicionalmente, se destaca la categoría de vivencia del disfrute del tiempo libre para dedicarse a ellos mismos y compartir sus experiencias con otros que pudieran comprenderlo.

Otra de las experiencias es volver a tener el control de su propia vida y darle sentido a la experiencia del cáncer. Esto es coincidente con otras investigaciones ${ }^{(47,48)}$ en que los participantes manifiestan mayor empoderamiento al elegir libremente el tratamiento porque de esta forma le dan sentido a una lucha activa contra el cáncer.

Aun cuando en este estudio se hace una mención especial a los proveedores que brindan apoyo y soporte en momentos difíciles, la literatura señala que desde la medicina tradicional aún existe escepticismo hacia las $\mathrm{TCA}^{(49)}$, argumentando que se necesitaría más evidencia empírica para que sean utilizadas en la práctica. Vinculado a esto están los estudios $^{(50,51)}$ que reafirman los hallazgos de esta revisión, en el sentido de que los prejuicios de los profesionales de salud transfieren inseguridades en la toma de decisión de las TCA y como respuesta, los pacientes no hablan de estas terapias con sus profesionales tratantes porque no ven el interés de éstos en ellas.

A pesar de esto, la OMS tiene una estrategia que promueve la utilización de la TCA, prestando apoyo a los estados miembros para que observen su contribución a la salud y promuevan su utilización segura y eficaz ${ }^{(52)}$. De igual forma, diversas organizaciones científicas de salud están generando evidencia para implementar su uso junto con la medicina convencional, a saber: El Centro Nacional de Salud Complementario e Integrativo de los Estados Unidos ${ }^{(53)}$ que realiza investigaciones sobre la utilidad y seguridad de las TCA; el Instituto de Investigación en Salud, de Australia ${ }^{(54)}$, que garantiza el acceso a evidencia confiable sobre medicamentos y tratamientos complementarios de amplio uso; la Asociación Británica de Medicina Complementaria, que cuenta con una red internacional de profesionales proveedores de terapias complementarias ${ }^{(55)}$ y la Sociedad Clínica de Oncología Australiana, que trabaja en educación, redes, defensa e investigación $^{(56)}$. Por su parte, las instituciones de salud son las llamadas a tomar esa evidencia e 
incorporarla en los protocolos de cuidados y los profesionales de salud, eslabón importante en este proceso, ya deben incorporar estos saberes desde los currículum que guiaron su formación. En particular, para enfermería es fundamental integrar cuidados basados en las $\mathrm{TCA}^{(57)}$ dado que aportan una dimensión que no se logra con la perspectiva biomédica. Lo anterior, implica utilizar las mejores evidencias, con diversos abordajes ${ }^{(58,59)}$ que demuestren ser las intervenciones más efectivas para el cuidado de las personas con cáncer. En este sentido, es importante avanzar en estudios experimentales, que midan los beneficios de las TCA reportados por la investigación cualitativa ${ }^{(21}$, ${ }^{32)}$, tal como ya lo están haciendo centros integrales contra el cáncer en Sydney ${ }^{(60)}$ y Corea ${ }^{(48)}$. En Chile se encuentra la experiencia de la Casa Bambú ${ }^{(61)}$, un centro de terapias complementarias y cuidado humanizado, que presenta una visión integrativa de la salud siguiendo las propuestas estratégicas de la OMS. Todos estos centros presentan muy buenos resultados, sin embargo, se necesita universalizar esta práctica de cuidado, aún no tradicional y poco frecuente.

Los resultados de este estudio mostraron que, aunque las TCA fueron de distinto tipo, la experiencia siempre fue favorable. Es conveniente realizar estudios que permitan conocer cuál es la más beneficiosa ${ }^{(21,28)}$, cuáles son las experiencias negativas de su uso y cuáles son las percepciones

\section{REFERENCIAS}

1. Organización Mundial de la Salud. Datos y cifras sobre el cáncer [Internet]. 2014 [citado 3 ago 2019]. Disponible en: https://www.who.int/cancer/about/ facts/es/

2. Ministerio de Salud de Chile. Plan nacional de Cáncer 2018-2028 [Internet]. 2018 [citado 3 ago 2019]. Disponible en: https://www.minsal.cl/wpcontent/uploads/2019/01/2019.01.23_PLANNACIONAL-DE-CANCER_web.pdf

3. Castiblanco R, Casas C, Carrillo A, Fuentes C, Melgarejo L. Prácticas alternativas al modelo de salud occidental utilizadas por cuidadores de pacientes con neoplasia hematológicas. Cul Cuid [Internet]. 2019 [citado 4 ago 2019]; 23(53): 168179. Disponible en: https://dialnet.unirioja.es/ servlet/articulo? codigo $=6919500$ y experiencias de los pacientes escépticos que, a pesar de ello, participan de las sesiones complementarias $^{(30)}$.

En el área de la formación profesional, enfermería debería incluir el conocimiento de las TCA en los planes de estudio y en investigación, debe realizar estudios de sus experiencias cotidianas, para evidenciar el aporte de las TCA a la salud de las personas.

\section{CONCLUSIÓN}

La evidencia científica de enfermería, relacionada con la experiencia de las personas con cáncer que practican terapias complementarias, señala que estas personas experimentan vivencias mayoritariamente positivas de las que emergen 6 categorías, relacionadas con las creencias de los beneficios y prejuicios que traen consigo y que están directamente relacionadas con las diferencias que ellos perciben entre las terapias tradicionales y complementarias. De igual forma, se evidencia una mirada holística de estas prácticas, integrada por las dimensiones física, psicológica, espiritual y social del ser, que genera en estos pacientes tiempo para el cuidado, amor personal, búsqueda del sentido de la experiencia del cáncer y entrega control personal de su vida.

4. FernándezA, PirisA, CabrerM, BarqueroA.Situación actual de las Terapias Complementarias en España en el Grado de Enfermería. Rev Latino-am Enfermagem [Internet]. 2013 [citado 4 ago 2019]; 21(3): 1-9. Disponible en: http://www.scielo.br/scielo. php?pid=S010411692013000300679\&script $=$ sci_ arttext\&tlng=es

5. Cano L. Correlación entre las búsquedas sobre terapias complementarias en Google y su uso por parte de la población española. Panace@ [Internet]. 2016 [citado 4 ago 2019]; 17(44): 124-132. Disponible en: https://www.tremedica.org/wpcontent/uploads/n44_tribuna-LCano-Oron.pdf

6. Ministerio de Salud de Chile. Anexo: Terapias Complementarias [Internet]. 2013 [citado 5 ago 2019]. Disponible en: http://www.sename. cl/wsename/p14_08-08-2017/Anexo-TerapiasComplementarias.pdf 
7. Smithson J, Paterson C, Britten N, Evans M, Lewith G. Cancer patients' experiences of using complementary therapies: polarization and integration. J Health Serv Res Policy [Internet]. 2010 [citado 5 ago 2019]; 15(2): 54-61. Disponible en: 10.1258/jhsrp.2009.009104

8. Ben-Arye E, Poper-Giveon A, Samuels N, Mutafoglu $\mathrm{H}$, Schiff E, Omran S, et al. Communication and integration: a qualitative analysis of perspectives among Middle Eastern oncology healthcare professionals on the integration of complementary medicine in supportive cancer care. J Cancer Res Clinical Oncology [Internet]. 2016 [citado 5 ago 2019]; 142(1):1117-1126. Disponible en: 10.1007/s00432-016-2120-9

9. Evans M, Shaw A, Sharp D, Thompson E, Falk S, Turton P, et al. Men with cancer: is their use of complementary and alternative medicine a response to needs unmet by conventional care?. Eur J Cancer Care (Engl) [Internet]. 2007 [citado 5 ago 2019]; 16: 517-525. Disponible en: 10.1111/j.13652354.2007.00786.x

10. Thomas T, Jackson V, Carlson H, Rinaldi S, Sousa A, Hansen A, et al. Communication differences between oncologists and palliative care clinicians: A qualitative analysis of early, integrated palliative care in patients with advanced cancer. J of Palliative Med [Internet]. 2019 [citado 7 ago 2019]; 22(1): 41-49. Disponible en: 10.1089/jpm.2018.0092

11. Binesh M, Reza M, Shirazi E, Oleson T, Hashem F. Comparison of Auricular Therapy with Sham in Children with Attention Deficit/Hyperactivity Disorder: A Randomized Controlled Trial. JACM [Internet]. 2020 [citado 9 jun 2019]; 1-6. Disponible en: http//doi.org/10.1089/ acm.2019.0477

12. Raffey M, Nikniaz Z, Farshi F, Sameni Z, Faramarzi E. Effects of Curcumin Supplementation on Quality of Life of Cystic Fibrosis Patients. IJP [Internet]. 2020 [citado 9 jun 2019]; 8(4): 169-176. Disponible en: 10.22038/IJP.2019.42990.3602

13. Liu L, Tan H, Yu S, Yin H, Baxter G. The effectiveness of tai chi in breast cancer patients: A systematic review and meta-analysis. Complement Ther Clin Pract [Internet]. 2020 [citado 9 jun 2020]; 38:101078. Disponible en: 10.1016/j. ctcp. 2019.101078

14. Irmak Z, Tanrıverdi O, Ödemiş H, Demir D. Use of Complementary and Alternative Medicine and Quality of Life of Cancer Patients Who Received Chemotherapy in Turkey. Complement Ther Med [Internet]. 2019 [citado 8 ago 2019]; 23(2): 1-31. Disponible en: https://doi.org/10.1016/j. ctim.2019.04.008

15. Than M, Anam A, Nurfarahi K, Asma' A, Hayati $\mathrm{M}$. Knowledge, use of complementary alternative medicine and health-related quality of life among cardiovascular disease patients. F Research [Internet]. 2019 [citado 8 ago 2019]; 3(5): 604616. Disponible en: https://www.scopus.com/ citation/output.uri?origin $=$ recordpage $\&$ view $=\& s r c$ =s\&eid=2-s2.0-85068402920\&output Type=expor tPdf

16. Fernández A, Salvador T. Formación y aplicación de las terapias complementarias en los cuidados de enfermería al paciente oncológico. Nursing [Internet]. 2010 [citado 8 ago 2019]; 28(7):5258. Disponible en: http://diposit.ub.edu/dspace/ handle/2445/34076

17. Crossetti M. Revisión integrativa de la investigación en enfermería, el rigor científico que se le exige. Rev Gaúcha Enferm [Internet]. 2012 [citado 10 ago 2019]; 33(2): 1011. Disponible en: http://www.scielo.br/scielo. php?pid=S198314472012000200002\&script $=$ sci arttex

18. Gálvez A. Lectura Crítica de un Estudio Cualitativo Interpretativo. Index Enferm [Internet]. 2003 [citado 9 jun 2019]; 42. Disponible en: http:// www.index-f.com/index-enfermeria/42revista/42 articulo_39-43.php

19. Salamonsen A, Kruse T, Eriksen S. Modes of Embodiment in Breast Cancer Patients Using Complementary and Alternative Medicine. Qual Health Res [Internet]. 2012 [citado 10 ago 2019]; 22(11): 1497-1512. Disponible en: https://journals. sagepub.com/doi/pdf/10.1177/1049732312457077

20. Legenne M, Chirac A, Ruer M, Reix F, Filbet $M$. Perception of naturopathy for female patients with metastatic gynecological cancer: A qualitative study. Palliat Sup Care [Internet]. 2015 [citado 10 ago 2019]; 13: 1663-1668. Disponible en: 10.1017/ S1478951515000553

21. Canales M, Geller B. Surviving Breast Cancer The Role of Complementary Therapies. Fam Community Health [Internet]. 2003 [citado 11 ago 2019]; 26(1): 11-24. Disponible en: http://pesquisa. bvsalud.org/portal/resource/es/mdl-12802124

22. Correa I, Clavarino A, Eastwood H. Surviving, Relieving, Repairing, and Boosting Up: Reasons for Using Complementary/Alternative Medicine among Patients with Advanced Cancer: A Thematic Analysis. J Palliat Med [Internet]. 2005 [citado 12 ago 2019]; 8(5): 953-961. Disponible en: https://www.liebertpub.com/doi/abs/10.1089/ jpm.2005.8.953

23. Spadacio C, Filice de Barros N. Terapêuticas Convencionais e Não Convencionais no Tratamento do Câncer: os Sentidos Das Práticas Religiosas. Interface (Botucatu) [Internet]. 2009 [citado 12 ago 2019]; 13(30): 45-52. Disponible en: http://www. scielo.br/scielo.php?script=sci_arttext\&pid=S141432832009000300005\&lang=es

24. Casas C. Prácticas de Medicina Tradicional y 
Complementaria en Pacientes con Neoplasias Hematológicas. Index Enferm [Internet]. 2017 [citado 12 ago 2019]; 26(1-2): 20-24. Disponible en: http://scielo.isciii.es/scielo.php?script=sci_ abstract\&pid $=$ S113212962017000100005

25. Ben E, Preis L, Barak Y, Samuels N. A collaborative model of integrative care: Synergy between Anthroposophic music therapy, acupuncture, and spiritual care in two patients with breast cancer. Complement Ther Med [Internet]. 2018 [citado 12 ago 2019]; 40: 195-197. Disponible en: https:// doi.org/10.1016/j.ctim.2018.04.002

26. Williams A, Bulsara C, Joske D, Petterson A, Nowak A, Bennett K. An Oasis in the Hospital The Perceived Benefits of a Cancer Support Center in a Hospital Setting Offering Complementary Therapies. J Holist Nurs [Internet]. 2014 [citado 15 ago 2019]; 32(4): 250-260. Disponible en: $10.1177 / 0898010114526951$

27. Wanchai A, Armer J, Steward B. Breast Cancer Survivors' Perspectives of Care Practices in Western and Alternative Medicine. Oncol Nurs Forum [Internet]. 2010 [citado 15 ago 2019]; 37(4): 494500. Disponible en: http://eds.a.ebscohost.com/eds/ pdfviewer/pdfviewer?vid=1\&sid=bd91963d-a0864117-9e77-cd179cc6647d\%40sessionmgr4006

28. Dunwoody L, Smyth A, Davidson R. Cancer patients' experiences and evaluations of aromatherapy massage in palliative care. Int J Palliat Nurs [Internet]. 2002 [citado 15 ago 2019]; 8(10): 497-504. Disponible en: 10.12968/ ijpn.2002.8.10.10696

29. Amichai T, Grossman M, Richard M. Lung cancer patients' beliefs about complementary and alternative medicine in the promotion of their wellness. Eur J Oncol Nurs [Internet]. 2012 [citado 16 ago 2019]; 16: 520-527. Disponible en:10.1016/j.ejon.2012.01.004

30. Sirisupluxana P, Sripichyakan K, Wonghongkul T, Sethabouppha H, Pierce P. The meaning of complementary therapy from the perspective of Thai women with breast cancer. Nurs Health Sci [Internet]. 2009 [citado 16 ago 2019]; 11: 64-70. Disponible en: 10.1111/j.14422018.2009.00432.x

31. Loudan A, Barnett T, Williams A. Yoga, Breast Cancer-Related Lymphoedema and WellBeing: A Descriptive Report of Women's Participation in a Clinical Trial. J Clin Nurs [Internet]. 2017 [citado 16 ago 2019]; 26(23-24): 4685-4695. Disponible en: 10.1111 /jocn. 13819

32. Keim J, Albrecht T, Steeves R, Danhauer S. Young Women's Experiences With Complementary Therapies During Cancer Described Through Illness Blogs. West J Nurs Res [Internet]. 2013 [citado 17 ago 2019]; 35(10): 1309-1324. Disponible en: 10.1177/0193945913492897
33. Kirshbaum M, Stead M, Bartys S. An exploratory study of reiki experiences in women who have cancer. Int $\mathrm{J}$ Palliat Nurs [Internet]. 2016 [citado 17 ago 2019]; 22(4): 166-172. Disponible en: http://apps.webofknowledge. $\mathrm{com} /$ full_record.do?product $=$ WOS\&search mode $=$ GeneralSearch \&qid $=4 \& S I D=5 \mathrm{E} 1 \mathrm{ZsD}$ FwQdd9VHh4QIU\&page $=4 \&$ doc $=39$

34. Huberty J, Eckert R, Larkey L, Gowin K, Mitchell J, Mesa R. Perceptions of Myeloproliferative Neoplasm Patients Participating in an Online Yoga Intervention: A Qualitative Study. Integr Cancer Ther [Internet]. 2018 [citado 17 ago 2019]; 17(4): 1150-1162. Disponible en: https://doi. org/10.1177/1534735418808595

35. Strauss A, Corbin J. Bases de la investigación cualitativa: Técnicas y procedimientos para desarrollar la teoría fundamentada. 2a Ed. Colombia: Editorial Universidad de Antioquia; 2002.

36. Bae K, Kim E, Kong J, Kim J, Park S, Joon Jun $\mathrm{H}$, et al. Integrative cancer treatment may have a survival benefit in patients with lung cancer: A retrospective cohort study from an integrative cancer center in Korea. Medicine [Internet]. 2019 [citado 24 ago 2019]; 98(26): 1-10. Disponible en: 10.1097/MD.0000000000016048

37. Than M, Anam A, Nurfarahi K, Asma A, Hayati M. Knowledge, use of complementary alternative medicine and health-related quality of life among cardiovascular disease patients. F Research [Internet]. 2019 [cited 24 ago 2019]; 3(5): 604616. Disponible en: 10.26656/fr.2017.3(5).118

38. Voiß P, Lange S, Paul A, Dobos G, Kümmel S. Komplementäre Therapien in der Onkologie. Gynäkologe [Internet]. 2019 [citado 22 ago 2019]; 52: 135-143. Disponible en: https://doi. org/10.1007/s00129-018-4365-7

39. Voiß P, Dobos D, Choi K, Lange S, Kümmel S, Paul A. Lebensstilfaktoren und Komplementärmedizin beim Mammakarzinom. Gynäkologe [Internet]. 2017 [citado 23 ago 2019]; 50: 525-532. Disponible en: 10.1007/s00129-017-4067-6

40. Gall A, Leske S, Adams J, Matthews V, Anderson $\mathrm{K}$, Lawler S, et al. Traditional and Complementary Medicine Use Among Indigenous Cancer Patients in Australia, Canada, New Zealand, and the United States: A Systematic Review. Integr Cancer Ther [Internet]. 2018 [cited 23 ago 2019]; 17(3): 568-581. Disponible en: https://doi. org/10.1177/1534735418775821

41. Bismark R, Chen H, Dy G, Gage E, Mahoney M. Complementary and alternative medicine use among patients with thoracic malignancies. Supp Care Can [Internet]. 2014 [citado 21 ago 2019]; 22: 1857-1866. Disponible en: 10.1007/s00520014-2144-0

42. Eckerd C. Complementary and Alternative 
Therapy Use in Breast Cancer: Notable Findings. J Christ Nurs [Internet]. 2013 [citado 21 ago 2019]; 30(4): 218-225. Disponible en: 10.1097/ CNJ.0b013e3182a297f4

43. Armstrong M, Flemming K, Kupeli N, Stone P, Wilkinson S, Candy B. Aromatherapy, massage and reflexology: A systematic review and thematic synthesis of the perspectives from people with palliative care needs. Palliat Med [Internet]. 2019 [citado 21 ago 2019]; 33(7): 757-769. Disponible en: $10.1177 / 0269216319846440$

44. Bishop F, Yardley L, Lewith G. Treat or Treatment: A Qualitative Study Analyzing Patients' Use of Complementary and Alternative Medicine. Am J Public Health [Internet]. 2008 [citado 22 ago 2019]; 98(9): 1700-1705. Disponible en: $10.2105 /$ AJPH.2007.110072

45. Canon L, Mendoza I, Moreno C. Perfil sociodemográfico del usuario de la homeopatía en España. Aten Primaria [Internet]. 2018 [citado 18 ago 2019]; 49(1): 1-7. Disponible en: https://doi. org/10.1016/j.aprim.2018.07.006

46. Markovic M, Manderson L, Wray N, Quinn M. Complementary medicine use by australian women with gynaecological cancer. Psycho-Oncology [Internet]. 2006 [citado 18 ago 2019]; 15: 209220. Disponible en: 10.1002/pon.936

47. Keene M, Heslop I, Sabesan S, Glass B. Complementary and alternative medicine use in cancer: A systematic review. Complement Ther Clin Pract [Internet]. 2019 [citado 20 ago 2019]; 35: 33-47. Disponible en: https://doi.org/10.1016/j. ctcp.2019.01.004

48. Rausch S, et al. Widespread Use of Complementary and Alternative Medicine (CAM) among Non-Hodgkin Lymphoma (NHL) Survivors. Leuk Lymphoma [Internet]. 2015 [citado 20 ago 2019]; 56(2): 434-439. Disponible en: 10.3109/10428194.2014.916803

49. Broom A, Adams J. Oncology clinicians' accounts of discussing complementary and alternative medicine with their patients. Health (London) [Internet]. 2009 [citado 25 ago 2019]; 13(3): 317336. Disponible en: 10.1177/1363459308101806

50. Wode K, Henriksson R, Sharp L, Stoltenberg A, Hök J. Cancer patients' use of complementary and alternative medicine in Sweden: a crosssectional study. BMC Complement Altern Med [Internet]. 2019 [citado 25 ago 2019]; 19(62): 2-11. Disponible en: https://doi.org/10.1186/s12906-019-2452-5

51. $\mathrm{Ku} \mathrm{C}$, Koo M. Association of distress symptoms and use of complementary medicine among patients with cancer. J Clin Nurs [Internet]. 2012 [citado 26 ago 2019]; 21(5-6): 736-744. Disponible en: https://doi.org/10.1111/j.13652702.2011.03884.x
52. Organización Mundial de la Salud. Estrategia de la OMS sobre medicina tradicional 2014-2023 [Internet]. 2013 [citado 26 ago 2019]. Disponible en: https://apps.who.int/medicinedocs/documents/ s21201es/s21201es.pdf

53. National Center for Complementary and Integrative Health. NCCIH Facts at a Glance and Mission [Internet]. 2019 [citado 26 ago 2019]. Disponible en: https://nccih.nih.gov

54. Health Research Institute. The Science of Integrative Medicine: About NICM [Internet]. 2019 [citado 26 ago 2019]. Disponible en: https://www.nicm. edu.au/about_us/about_NICM

55. British Complementary Medicine Association BCMA. The International Network of Practitioners Promoting Professionalism. Prot Public [Internet]. 2019 [citado 27 ago 2019]. Disponible en: https:// www.bcma.co.uk/

56. Braun L, Harris J, Katris P, Cain M, Dhillon H, Koczwara B, et al. Clinical Oncology Society of Australia position statement on the use of complementary and alternative medicine by cancer patients. Asia Pac J Clin Oncol [Internet]. 2014 [citado 27 ago 2019]; 10: 289-296. Disponible en: 10.1111/ajco.12227

57. Andrade Y, Nascimento C, Assis Y, Salvador P, Tourinho F, Santos V. Práticas integrativas e complementares no tratamento oncológico e o papel da enfermagem. J res fundam care [Internet]. 2015 [citado 28 ago 2019]; 7(4): 3163-3174. Disponible en: https://dialnet.unirioja.es/servlet/ articulo?codigo $=5204803$

58. Huberty J, Eckert R, Larkey L, Joeman L, Mesa R. Experiences of Using a Consumer-Based Mobile Meditation App to Improve Fatigue in Myeloproliferative Patients: Qualitative Study. JMIR Cancer [Internet]. 2019 [citado 30 ago 2019]; 5(2): 1-15. Disponible en:10.2196/14292

59. Fitzsimmonsa A, Vollmer D, Bergeron C, Smith K, Patel A, Ory M, et al. Impact of complementary and alternative medicine offerings on cancer patients' emotional health and ability to self-manage health conditions. Complement Ther Med [Internet]. 2019 [citado 1 sep 2019]; 43: 102-108. Disponible en: https://doi.org/10.1016/j.ctim.2019.01.011

60. Grant S, Marthick M, Lacey J. Establishing an integrative oncology service in the Australian healthcare setting the Chris O'Brien Lifehouse Hospital experience. Support Care Cancer [Internet]. 2018 [cited 3 sep 2019]; 27(6): 20692076. Disponible en: https://doi.org/10.1007/ s00520-018-4460-2

61. Abad M, Urbina M, Cabrera M. Casa Bambú: Centro de Terapias Complementarias y Cuidado Humanizado [Internet]. 2019 [citado 3 sep 2019]. Disponible en: https://casabambu.cl/ 